\title{
\begin{tabular}{l|l} 
Mibraries & DSpace@MIT
\end{tabular}
}

\author{
MIT Open Access Articles
}

Electrochemical Windows of Room-Temperature Ionic Liquids from Molecular Dynamics and Density Functional Theory Calculations

The MIT Faculty has made this article openly available. Please share how this access benefits you. Your story matters.

Citation: Ong, Shyue Ping et al. "Electrochemical Windows of Room-Temperature lonic Liquids from Molecular Dynamics and Density Functional Theory Calculations." Chemistry of Materials 23.11 (2011): 2979-2986. Web.

As Published: http://dx.doi.org/10.1021/cm200679y

Publisher: American Chemical Society

Persistent URL: http://hdl.handle.net/1721.1/71594

Version: Author's final manuscript: final author's manuscript post peer review, without publisher's formatting or copy editing

Terms of Use: Article is made available in accordance with the publisher's policy and may be subject to US copyright law. Please refer to the publisher's site for terms of use. 


\title{
Electrochemical Windows of Room-Temperature
}

\section{Ionic Liquids from Molecular Dynamics and Density Functional Theory Calculations}

Shyue Ping Ong, ${ }^{\dagger}$ Oliviero Andreussi, ${ }^{\ddagger}$ Yabi Wu,${ }^{\dagger}$ Nicola Marzari, ${ }^{\ddagger}$ and Gerbrand Ceder*, $^{*}$

Department of Materials Science and Engineering, Massachusetts Institute of Technology, 77 Massachusetts Ave, Cambridge, MA 02139, and Department of Materials, University of Oxford, Parks Road, Oxford OXI 3PH, UK

E-mail: gceder@mit.edu

\begin{abstract}
We investigated the cathodic and anodic limits of six room-temperature ionic liquids (ILs) formed from a combination of two common cations - 1-butyl-3-methylimidazolium (BMIM) and N,N-propylmethylpyrrolidinium $(\mathrm{P} 13)$ - and three common anions $-\mathrm{PF}_{6}, \mathrm{BF}_{4}$ and bis(trifluoromethylsulfonyl)imide (TFSI) - using an approach that combines molecular dynamics (MD) simulations and density functional theory (DFT) calculations. All inter-ion interactions were taken into account by explicitly modeling the entire liquid structure using classical MD, followed by DFT computations of the highest occupied molecular orbital (HOMO) and lowest unoccupied molecular orbital (LUMO) energies. The relative cathodic and anodic limits of $\mathrm{BMIM} \mathrm{PF}_{6}, \mathrm{BMIM} \mathrm{BF}_{4}, \mathrm{BMIM}$ TFSI and P13 TFSI obtained from our approach are

\footnotetext{
${ }^{*}$ To whom correspondence should be addressed

${ }^{\dagger}$ Massachusetts Institute of Technology

†niversity of Oxford
} 
in fairly good agreement with existing experimental data. From our DFT calculations, we also obtained the cation- and anion-projected density of states (DOS), which provides information on the likely species contributing to reductive and oxidative decomposition. Our predictions support Howlett et al.'s earlier finding ${ }^{1}$ that the TFSI anion is less stable than the P13 cation against reduction. In addition, our results provide some surprising evidence of possible cation anodic instability; we predict the aromatic BMIM cation to be less stable against oxidation than the respective anions in $\mathrm{BMIM} \mathrm{PF}_{6}$ and $\mathrm{BMIM} \mathrm{BF}_{4}$, and the $\mathrm{P} 13$ cation to be less stable against oxidation than the $\mathrm{PF}_{6}$ anion in $\mathrm{P}_{13} \mathrm{PF}_{6}$. We also present a comparison of the predictions of our approach with that of simpler approximations based on in vacuo or polarizable continuum model calculations.

Keywords: electrochemical windows, molecular dynamics, density functional theory, roomtemperature, ionic liquids, polarizable continuum model, imidazolium, pyrrolidinium, $P F_{6}, B F_{4}$, bis(trifluoromethylsulfonyl)imide

Shyue Ping Ong, Oliviero
Andreussi, Yabi Wu, Nicola
Marzari and Gerbrand Ceder*
Chem. Mater. 2011, XX, YYYY
Electrochemical Windows of
Room-Temperature Ionic Liquids
from Molecular Dynamics and
Density Functional Theory
Calculations

Combined molecular dynamics simulations and density functional theory calculations provide quantitative predictions of electrochemical windows and point to hitherto unsuspected cation oxidative instability in common room-temperature ionic liquids based on the pyrrolidium and imiadzolium cations and the PF6, BF4 and bis-(trifluoromethylsulfonyl)imide (TFSI) anions.

\section{Introduction}

The electrochemical window of a solvent is an important design criteria for electrochemical applications. An electrochemical solvent, such as the electrolyte in a lithium-ion battery or supercapacitor, must support the voltage in which the electrochemical device operates. The electrochemical window is defined as the difference between the cathodic and anodic limits, which are the poten- 
tials at which reduction and oxidation of the solvent take place respectively. For certain applications (e.g., supercapacitors), it is the overall window that matters, while in other applications, the actual cathodic and anodic limits relative to some reference is the limiting factor. For example, in lithium-ion batteries where the anode potential is set by $\mathrm{Li} / \mathrm{Li}^{+}$, the cathodic limit relative to $\mathrm{Li} / \mathrm{Li}^{+}$would determine whether the solvent would be reduced by lithium metal, and the anodic limit would determine the voltage allowable for the cathode.

In the pursuit of higher energy density storage systems, the electrochemical windows of current electrolytes have become a limiting factor. For example, the typical organic carbonate electrolyte used in current lithium-ion batteries, a mixture of ethylene carbonate (EC) and dimethyl carbonate (DMC) with $\mathrm{LiPF}_{6}$ added to improve $\mathrm{Li}^{+}$conductivity, can only support a voltage up to approximately $5 \mathrm{~V}^{2}$ Furthermore, this EC/DMC electrolyte is unstable against Li in the graphitic carbon anode and works only because of the formation of a passivating solid-electrolyte interphase (SEI) layer that prevents further reaction.

Room-temperature ionic liquids (ILs) have garnered increasing interest as electrolytes in electrochemical applications. ${ }^{3-9}$ As a class of materials, ILs generally exhibit low volatility, low flammability and high thermal stability, which provides significant safety advantages over flammable organic compounds. This is especially important in the application of Li-batteries beyond smallscale portable electronics to large-scale applications such as hybrid electric vehicles (HEVs) and electric vehicles. Many ILs also have wide electrochemical windows of approximately 5-6 V or more, ${ }^{10,11}$ which are considerably larger than that of current organic electrolytes. A more electrochemically stable electrolyte could unlock the potential of high-voltage cathodes with higher power density. For example, $\mathrm{LiNiPO}_{4}$, which is predicted to have a potential greater than $5 \mathrm{~V},{ }^{12}$ would have $50 \%$ higher energy density than the Fe-based $\mathrm{LiFePO}_{4}$ system currently under development for HEVs. Similarly, $\mathrm{Li}\left(\mathrm{Ni}_{0.5} \mathrm{Mn}_{1.5}\right) \mathrm{O}_{4}$ has higher capacity, higher voltage and better rate capability than the Mn-based spinel currently in use for large format batteries, but operates at a high voltage close to the breakdown of the carbonate electrolyte. ${ }^{13}$

In ILs, it has typically been assumed that the cathodic limit is set by the reduction of the 
cations and the anodic limit is set by the oxidation of the anions. ${ }^{14,15}$ However, recent experiments have demonstrated that this may not always be the case. For instance, experimental and theoretical investigations by Howlett et al. ${ }^{1}$ have provided evidence that the commonly used bis(trifluoromethylsulfonyl)imide (TFSI) undergoes reductive decomposition at a potential that is more than $1 \mathrm{~V}$ above the potential at which the $\mathrm{N}, \mathrm{N}$-propylmethylpyrrolidinium (P13) cation decomposes.

In this work, we investigated the cathodic and anodic limits of six common ILs using an approach that combines molecular dynamics simulations and density functional theory calculations. ${ }^{16}$ In our approach, all inter-ion interactions are taken into account by explicitly modeling the entire liquid structure, which is in contrast to previous approaches to calculating solvent electrochemical windows that focused primarily on isolated molecules or ion-pairs in vacuo or in a

continuum solvent. ${ }^{17-24}$ In addition, our approach also provides information on the species (cation or anion) contributing to the redox stability of the overall liquid. We show that the predictions from our approach supports the previous finding that the TFSI anion is less stable than the P13 cation against reduction. Our results also provide some surprising evidence that points to possible cation anodic instability, especially in the case of the aromatic 1-butyl-3-methylimidazolium (BMIM) cation. We compare the results obtained using this approach to the predictions of simpler approximations based on in vacuo and polarizable continuum model (PCM) calculations, and discuss the limitations of each level of approximation. The approach used in this work can potentially be used to estimate the electrochemical windows of other solvents as well.

\section{Methodology}

\section{Estimating cathodic and anodic limits of common ILs}

We investigated all possible combinations of two common IL cations, 1-butyl-3-methylimidazolium (BMIM) and N,N-propylmethylpyrrolidinium (P13), and three common IL anions, $\mathrm{PF}_{6}, \mathrm{BF}_{4}$ and bis(trifluoromethylsulfonyl)imide (TFSI), i.e., a total of six different ILs (see Figure 1). These 
ions were chosen because of the widely available experimental data for $\mathrm{BMIM} \mathrm{PF}_{6}, \mathrm{BMIM} \mathrm{BF}_{4}$, BMIM TFSI and P13TFSI, as well as to study a diverse range of local environments, e.g., the more charge-delocalized aromatic BMIM cation and TFSI anion versus the more charge-localized aliphatic $\mathrm{P} 13$ cation and $\mathrm{PF}_{6}$ and $\mathrm{BF}_{4}$ anions.

To our knowledge, there is no reported experimental data for $\mathrm{P}_{13} \mathrm{PF}_{6}$ and $\mathrm{P} 13 \mathrm{BF}_{4}$ in the literature. ILs based on the similar tetraethylammonium cation $+\mathrm{BF}_{4}$ or $\mathrm{PF}_{6}$ have been reported to have fairly high melting points above room temperature, ${ }^{25}$ and it is unclear whether the P13 analogues are liquids at room-temperature. We have included $\mathrm{P} 13 \mathrm{PF}_{6}$ and $\mathrm{P} 13 \mathrm{BF}_{4}$ in our investigations only for the sake of completeness and to ascertain general trends in the electrochemical windows with changes in cation or anion.

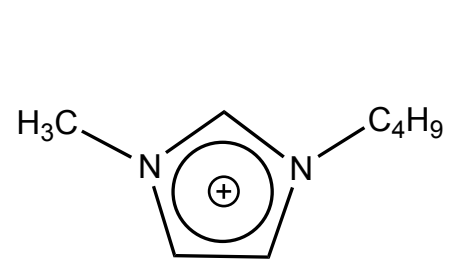

(a) BMIM

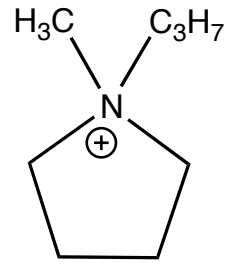

(b) P13

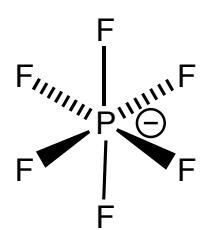

(c) $\mathrm{PF}_{6}$

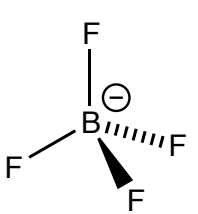

(d) $\mathrm{BF}_{4}$

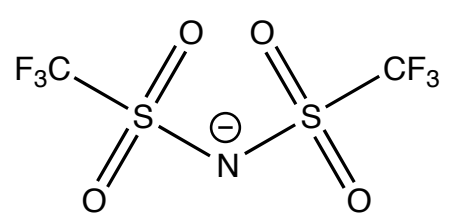

(e) TFSI

Figure 1: IL ions investigated. Cations: (a) 1-butyl-3-methylimidazolium (BMIM); (b) N,Npropylmethylpyrrolidinium (P13). Anions: (c) Hexafluorophosphate $\left(\mathrm{PF}_{6}\right)$; (d) Tetrafluoroborate $\left(\mathrm{BF}_{4}\right)$; (e) bis(trifluoromethylsulfonyl)imide (TFSI).

We assume that the cathodic limit is set by the lowest unoccupied molecular orbital (LUMO) and the anodic limit is set by the highest occupied molecular orbital (HOMO). The potentials of the cathodic and anodic limits, $V_{\mathrm{CL}}$ and $V_{\mathrm{AL}}$, for a one-electron transfer can then be obtained from the respective orbital energies using the following expressions:

$$
V_{\mathrm{CL}}=-\frac{\varepsilon_{\mathrm{LUMO}}}{e}
$$




$$
V_{\mathrm{AL}}=-\frac{\varepsilon_{\mathrm{HOMO}}}{e}
$$

where $\varepsilon_{\text {номо }}$ and $\varepsilon_{\mathrm{Lumo}}$ are the energies of the HOMO and LUMO levels respectively, and $e$ is the electron charge.

For the six ILs chosen, we compared the cathodic and anodic limits predicted using three levels of approximations.

The simplest level of approximation is to calculate the electron affinities (EA) and ionization energies (IE) of each of the constituent ions individually in vacuo using the $\Delta$-SCF approach, i.e., by explicitly taking the difference between the calculated energy of an ion and the calculated energy of that ion with one additional or one less electron. ${ }^{26}$ The EA corresponds to the energy of the LUMO level, while the IE corresponds to the energy of the HOMO level. For an IL formed from a combination of a cation $\mathrm{C}$ and anion $\mathrm{A}$, we assume that the cathodic limit is given by the maximum of the cathodic limits of the two ions, and the anodic limit is given by the minimum of the anodic limits of the two ions, i.e.,

$$
\begin{aligned}
& V_{\mathrm{CL}}^{\text {vacuum }}=\max \left(V_{\mathrm{CL}, \mathrm{C}}^{\text {vacuum }}, V_{\mathrm{CL}, \mathrm{A}}^{\text {vacum }}\right) \\
& V_{\mathrm{AL}}^{\text {vacuum }}=\min \left(V_{\mathrm{AL}, \mathrm{C}}^{\text {vacum }}, V_{\mathrm{AL}, \mathrm{A}}^{\text {vacuum }}\right)
\end{aligned}
$$

where $V_{\mathrm{CL}, \mathrm{i}}^{\mathrm{vacuum}}$ and $V_{\mathrm{AL}, \mathrm{i}}^{\mathrm{vacum}}$ are the in vacuo cathodic and anodic limits of ion i respectively.

The next level of approximation is to model each ion in solution using a continuum solvation model, and calculate the HOMO and LUMO levels in solution. ${ }^{27,28}$ In this work, we have adopted the integral equation formalism variant of the polarizable continuum model (PCM). ${ }^{29-46}$ This model takes into account, in mean-field manner, the stabilization of an ion from the dielectric response of its surroundings. Similar to the vacuum calculations, we assume the following:

$$
\begin{aligned}
& V_{\mathrm{CL}}^{\mathrm{PCM}}=\max \left(V_{\mathrm{CL}, \mathrm{C}}^{\mathrm{PCM}}, V_{\mathrm{CL}, \mathrm{A}}^{\mathrm{PCM}}\right) \\
& V_{\mathrm{AL}}^{\mathrm{PCM}}=\min \left(V_{\mathrm{AL}, \mathrm{C}}^{\mathrm{PCM}}, V_{\mathrm{AL}, \mathrm{A}}^{\mathrm{PCM}}\right)
\end{aligned}
$$


The highest level of approximation is to calculate the HOMO and LUMO levels of the six ILs using a combination of molecular dynamics (MD) simulations and periodic boundary condition (PBC) density functional theory (DFT) calculations on unit cells containing the IL cations and anions in their liquid configuration. These calculations were carried out in two steps:

1. Each IL was first equilibrated via MD simulations using well-established force-fields. From the MD simulations, we extracted 10 equilibrated snapshots spaced $1000 \mathrm{MD}$ steps $(\approx 2 \mathrm{ps}$ of simulation time) apart for each IL system.

2. The snapshots were then used as inputs for periodic boundary DFT calculations to determine the density of states (DOS) and the HOMO and LUMO energies at the MD-equilibrated geometry, i.e., no further geometry optimization was performed. The HOMO and LUMO energies of each IL is then obtained as the average of the HOMO and LUMO energies of all 10 snapshots of each IL, and aligned to a common vacuum reference using its Hartree potential.

\section{Computational methods}

\section{Molecular dynamics simulations}

Our classical molecular dynamics (MD) simulations were carried out using the DLPOLY program. ${ }^{47-49}$ For the interaction potentials, the anions and the BMIM cation were modeled using the OPLS-AA ${ }^{50}$ based force field derived by Lopes et al. ${ }^{51-53}$ The force field for the P13 cation was derived from the standard OPLS parameters for amines, ${ }^{50,53}$ while atomic charges were explicitly parametrized using the CHelpG procedure ${ }^{54}$ on ab initio MP2/cc-pVTZ(-f) calculations. Equilibration of the systems in the NPT ensemble was enforced using the Berendsen thermostat

and barostat. ${ }^{55}$ A simulation time step of $2 \mathrm{fs}$ and an interaction cutoff radius of $15 \AA$ were used throughout.

Initial configurations containing 128 ion pairs were generated starting from a face-centered cubic lattice with the ions occupying random lattice sites. Equilibration runs were performed at 
$500 \mathrm{~K}$ and zero pressure until convergence of the statistical average of the density was achieved. Following equilibration, configurations of clusters of 16 ion pairs were extracted from the final configuration of the trajectories. These smaller clusters were then equilibrated with periodic boundary conditions at $500 \mathrm{~K}$ and zero pressure, followed by quenching for a few nanoseconds at $300 \mathrm{~K}$, to obtain the input structures for the DFT calculations.

\section{Periodic boundary condition DFT calculations}

The DOS calculations were performed using the Perdew-Burke-Ernzerhof (PBE) generalized gradient approximation functional (GGA) with an energy cutoff of $500 \mathrm{eV}$. Projected augmented wave $(\mathrm{PAW})^{56}$ pseudopotentials were used, as implemented in the the Vienna ab initio simulation package (VASP). ${ }^{57}$ Owing to the large size of the unit cells, only a single $\Gamma k$-point was used, and our convergence tests found that increasing $k$-point density to a larger $\Gamma$-centered $2 \times 2 \times 2 \operatorname{grid}$ had a negligible effect on the orbital energies obtained. All calculations were non-spin-polarized.

To align the orbital energies and density of states obtained for all ILs to a common reference,

we determined the Hartree potential for all ILs relative to a vacuum layer. ${ }^{58-60}$ A vacuum slab of equal size was attached to each IL unit cell in the [100] direction. Using this extended unit cell, we then calculated the Hartree potential using the same parameters as that used for the DOS computations. The average Hartree potential is then plotted as a function of the cell $a$-coordinate (see Supporting Information) to determine the difference in the average Hartree potentials in the IL and vacuum regions.

For each IL, only one Hartree potential shift relative to vacuum was determined using the MD configuration that exhibited the smallest dipole between the two slab surfaces, i.e., the IL + vacuum cell that had the flattest Hartree potential in the vacuum region was used. We kept a constant shift for each IL as the difference between the average Hartree potential in the IL region and in the vacuum region. The interfacial regions between the IL and vacuum where there are sharp changes in the Hartree potential were excluded in calculating the average Hartree potentials. The constant shift was then applied to the orbital energies and DOS for the IL. The calculated Hartree potential 
shifts for the six ILs are given in Table 1.

Table 1: Hartree potential shifts

\begin{tabular}{lc}
\hline Ionic Liquid & Shift $\left(E_{\text {Hartree }}^{\mathrm{IL}}-E_{\text {Hartree }}^{\text {vacum }}\right)$ \\
\hline BMIM PF $_{6}$ & $-5.07 \mathrm{eV}$ \\
BMIM BF $_{4}$ & $-4.83 \mathrm{eV}$ \\
BMIM TFSI & $-5.66 \mathrm{eV}$ \\
P13 PF $_{6}$ & $-5.11 \mathrm{eV}$ \\
P13 $\mathrm{BF}_{4}$ & $-4.56 \mathrm{eV}$ \\
P13 TFSI & $-5.42 \mathrm{eV}$ \\
\hline
\end{tabular}

\section{Vacuum and polarizable continuum calculations}

All vacuum and polarizable continuum model calculations in this paper were performed using the Gaussian 03 quantum chemistry package. ${ }^{61}$ Geometry optimizations were carried out at the B3LYP/6-31+G(d) level and were followed by single-point energy calculations at the B3LYP/6$311+\mathrm{G}(2 \mathrm{~d}, \mathrm{p})$ level. The hybrid B3LYP density functional based on Becke's three-parameter exchange functional ${ }^{62}$ and the correlation functional of Lee, Yang and Parr ${ }^{63}$ was chosen as it has been shown to provide good accuracy for EAs and IEs at a reasonable computation cost. ${ }^{64}$ The inclusion of diffuse functions in the basis sets ensures an adequate description of the diffuse electron cloud of anions and the radicals formed from cation reduction. Closed-shell restricted wave function calculations were used in the treatment of the cations and anions, while unrestricted open-shell wavefunctions were used for the ions that have singly occupied orbitals. Frequency analysis was performed to ensure that structures obtained were minimum energy structures rather than transition structures, and also to obtain the thermochemical corrections for the Gibbs free energy. The thermochemical corrections were then scaled using the factor of 0.9806 determined earlier by Scott $e t$ al. ${ }^{65}$ for the B3LYP/6-31G(d) model chemistry.

All PCM calculations were performed using a dielectric constant of 12, which is typical of ILs. $^{66}$ 


\section{Results}

\section{Electrochemical windows from MD + DFT calculations}

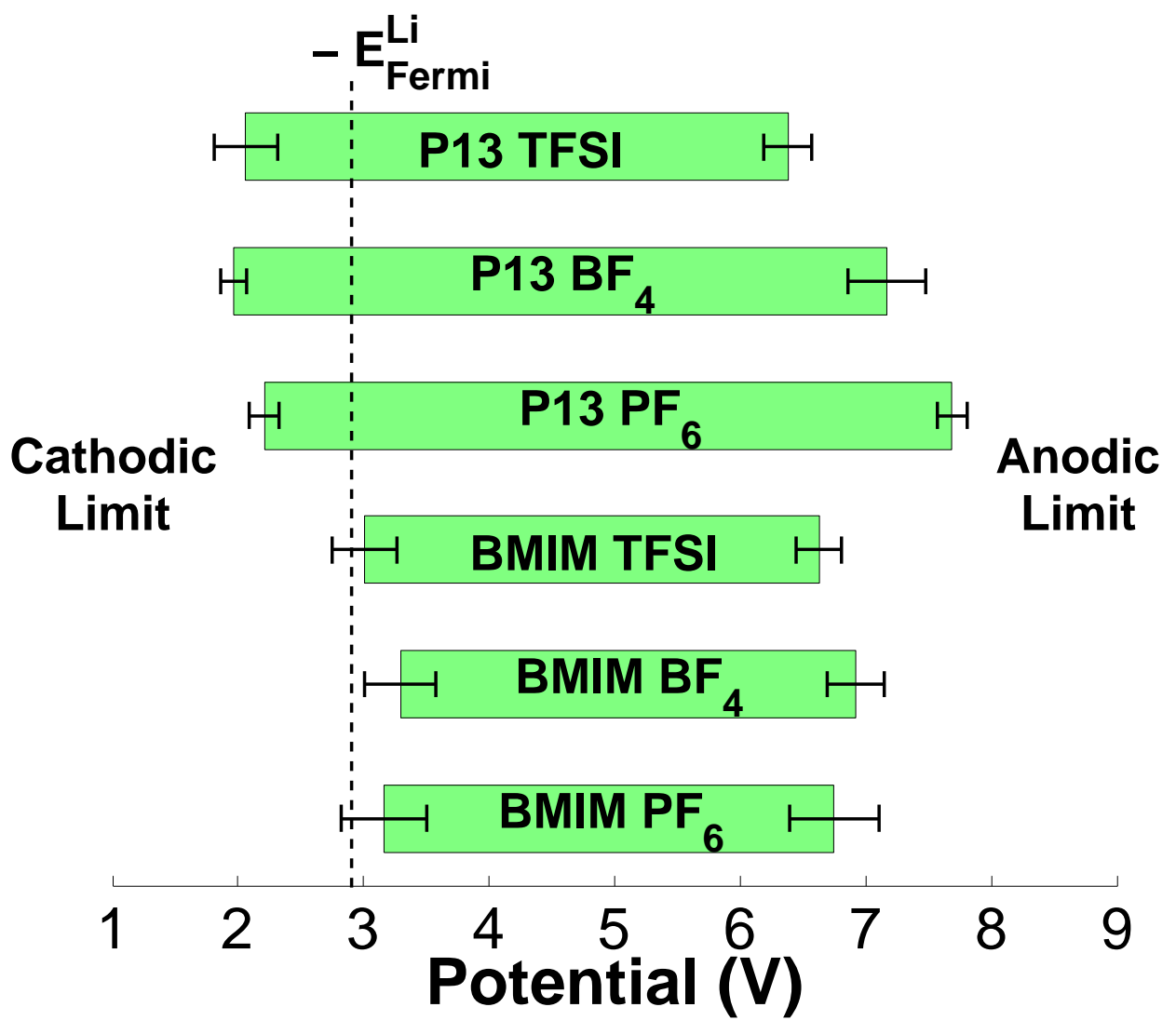

Figure 2: Electrochemical windows of ILs from MD + DFT calculations. Solid green bars indicate the potential range for which the IL is stable. The calculated lithium metal Fermi level is also indicated. The $95 \%$ confidence interval for each limit is given by the black dumbbells. The potential scale is relative to the vacuum level.

Figure 2 summarizes the calculated electrochemical windows of the six ILs from our MD + DFT calculations. The solid green rectangular bars indicate the potential region for which a particular IL is stable. The minimum of the stable potential region is the cathodic limit (where reduction of the IL is expected to occur), while the maximum is the anodic limit (where oxidation 
of the IL is expected to occur). As the limits were determined as the average of $10 \mathrm{MD}$ snapshots for each IL, the $95 \%$ confidence interval for each limit is indicated by the black dumbbells. In the figure, we also plotted the calculated Fermi level of lithium metal on the same potential scale using the same Hartree potential alignment approach. Our calculated value for - $\mathrm{E}_{\mathrm{Fe}}^{\mathrm{Li}}$. of 2.91 $\mathrm{eV}$ (relative to vacuum) is in excellent agreement with the experimental work function of lithium of $2.93 \mathrm{eV},{ }^{67}$ which lends further support for our Hartree potential approach to aligning the orbital energies and DOS.

Before we outline the key observations from our results, we would like to note that comparing the predictions from our calculations with experiments is complicated by the fact that measured electrochemical windows depend heavily on the measurement conditions. ${ }^{10,15}$ Typically, the electrochemical window is determined by performing a linear sweep voltammetry using inert electrodes (e.g., Pt or Au) and measuring the cathodic and/or anodic currents, which are indicative of electrolyte reduction or oxidation. However, reported electrochemical windows in the literature vary widely in the measurement conditions, differing in the type of electrodes, the arbitrary current cut-off used to determine the onset of redox processes (typically between 0.1 and $1.0 \mathrm{~mA}$ $\mathrm{cm}^{-2}$ ) and the references used, some of which may not be strictly electrochemically defined. In the case of ILs, this difficulty is further compounded by their sensitivity to water, air and other impurities. ${ }^{68-70}$

Notwithstanding the above, a few consistent observations can be made from Figure 2 and experimental data from studies comparing several ILs:

1. Relative cathodic stability of P13- and BMIM-based ILs. The P13-based ILs have significantly lower cathodic limits than the BMIM-based ILs, in agreement with the general experimental observation that aliphatic cations such as P13 are in general more stable than aromatic ones such as BMIM. ${ }^{10,14,15}$

2. Relative anodic stability. For the anodic limits, a clear trend of $\mathrm{PF}_{6}>\mathrm{BF}_{4}>\mathrm{TFSI}$ is observed for the P13-based ILs, which is in agreement with the relative anion stabilities calculated by Ue et al. ${ }^{18}$ and our previous work. ${ }^{23}$ On the other hand, all BMIM-based ILs 
have relatively similar anodic limits, regardless of the anion. As we shall demonstrate in the following subsection, we hypothesize that this is due to the relative instability of BMIM towards both reduction and oxidation.

3. Underestimation of electrochemical windows. The predicted electrochemical windows for the BMIM-based ILs are all around 3.6 V, which is somewhat smaller than the 4.2-4.6 V observed by Lewandowski et al. ${ }^{71}$ using Pt working electrodes in a $\mathrm{AglAg}^{+}$in DMSO reference. However, it is well-known that DFT based on the local density approximation (LDA) and GGA underestimates the energy gap between occupied and unoccupied states by $30 \%$ $100 \%{ }^{72-75}$ Indeed, using the the Heyd-Scuseria-Ernzerhof hybrid functional (HSE06), ${ }^{76,77}$ which is well-known to provide more accurate band gaps, ${ }^{75,78}$ we calculated the electrochemical windows of $\mathrm{BMIM} \mathrm{PF}_{6}, \mathrm{BMIM} \mathrm{BF}_{4}, \mathrm{BMIM}$ TFSI and P13 TFSI (the four experimentally known ILs among the six we investigated) for a representative liquid configuration for each IL to be approximately $4.9 \mathrm{~V}, 5.1 \mathrm{~V}, 5.5 \mathrm{~V}$ and $6.1 \mathrm{~V}$ respectively, significantly larger than the GGA electrochemical windows (see Figure 3). However, the HSE06 functional is far too expensive for the purposes of our investigations, which involve multiple calculations on unit cells containing more than 600 atoms in some cases. Despite this limitation of GGA, we observe that the relative trends in the limits are qualitatively the same in both HSE06 and GGA. In general, the electrochemical windows of all three BMIM-based ILs are fairly similar both in experiments and in our calculations.

4. Electrochemical window of P13 TFSI. Our calculations predict the electrochemical window of P13 TFSI to be significantly wider than the BMIM-based ILs by approximately $0.7 \mathrm{~V}$. This prediction disagrees with Lewandowski et al.'s measurements, ${ }^{71}$ which found the electrochemical window of P13 TFSI to be $3.7 \mathrm{~V}$ and somewhat smaller than that of the BMIM-based ILs. However, a more recent work by Lewandowski et al. ${ }^{79}$ found the electrochemical window (on glassy carbon) of 0.1 M LiTFSI dissolved in the very similar N-N-butylmethylpyrrolidinium TFSI IL to be $5.1 \mathrm{~V}$, slightly wider than the electrochemical 
window of $4.9 \mathrm{~V}$ measured for $0.1 \mathrm{M} \mathrm{LiPF}_{6}$ in $\mathrm{BMIM} \mathrm{PF}_{6}$. This finding is in qualitative agreement with our predictions. Experiments by Barisci et al. ${ }^{80}$ using Pt working electrodes and $\mathrm{Ag}$ wire reference also found the electrochemical window of P13 TFSI $(5.7 \mathrm{~V})$ to be larger than that of BMIM PF 6 (3.2 V). It should be noted, however, that Barisci et al. measured P13 TFSI to have a higher anodic limit and lower cathodic limit than $\mathrm{BMIM} \mathrm{PF}_{6}$, while our calculations predict P13 TFSI to have both lower cathodic and anodic limits than BMIM PF 6 .

5. Stability versus lithium. The cathodic limits of the BMIM ILs are all above the Li potential, which indicates that BMIM is likely to be reduced by lithium metal. The P13 ILs, on the other hand, have cathodic limits that are below the Li potential, which suggest that they could be stable against lithium. This is consistent with the findings of Fernicola et al. ${ }^{81}$ who found that a mixture of the slightly different N-N-butylethylpyrrolidnium TFSI with Li TFSI to be stable with lithium for at least a week of contact. However, there are conflicting reports on this matter, such as the work of Lewandowski et al. ${ }^{79}$ who found that only piperidiniumbased ILs have cathodic limits that are below the $\mathrm{LilSEI} / \mathrm{Li}^{+}$potential.

\section{Calculated density of states for ILs}

Figure 3 shows representative plots of the projected DOS of the six ILs by cation and anion contributions. The cation- and anion-projected DOS allows us to determine the species in the IL that contributes to the HOMO (cathodic limit) and LUMO (anodic limit) levels. From the figures, we can make the following observations:

1. Cathodic instability of TFSI in P13 TFSI. For P13 TFSI, the LUMO is dominated by the anion, which demonstrates that anion reductive stability could be an issue. This observation is in agreement with the experimental and theoretical work of Howlett et al., ${ }^{1}$ who showed that it is the TFSI anion that determines the cathodic limit in P13 TFSI.

2. Possible cathodic instability of $\mathbf{P F}_{6}$ in $\mathbf{P} 13 \mathbf{P F}_{6}$. For $\mathrm{P}_{13} \mathrm{PF}_{6}$, our calculated DOS shows 


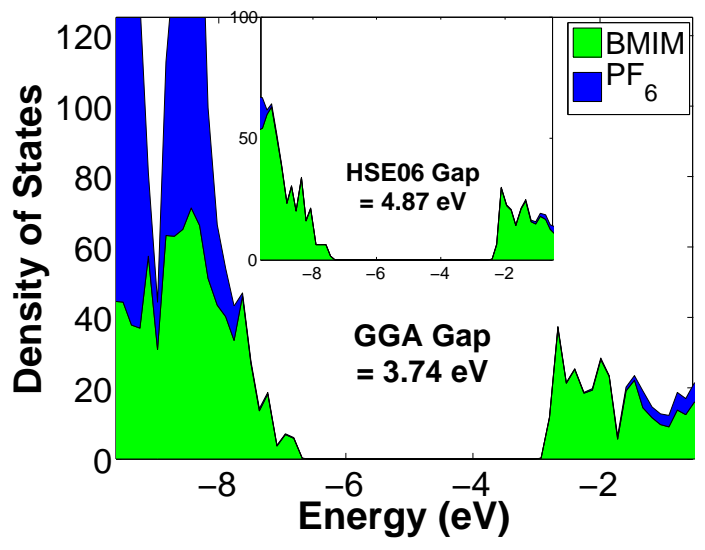

(a) $\mathrm{BMIM} \mathrm{PF}_{6}$

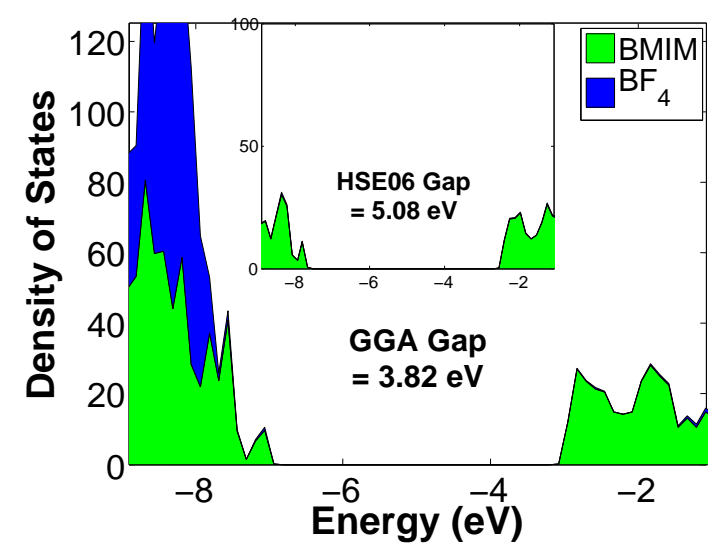

(c) $\mathrm{BMIM} \mathrm{BF}_{4}$

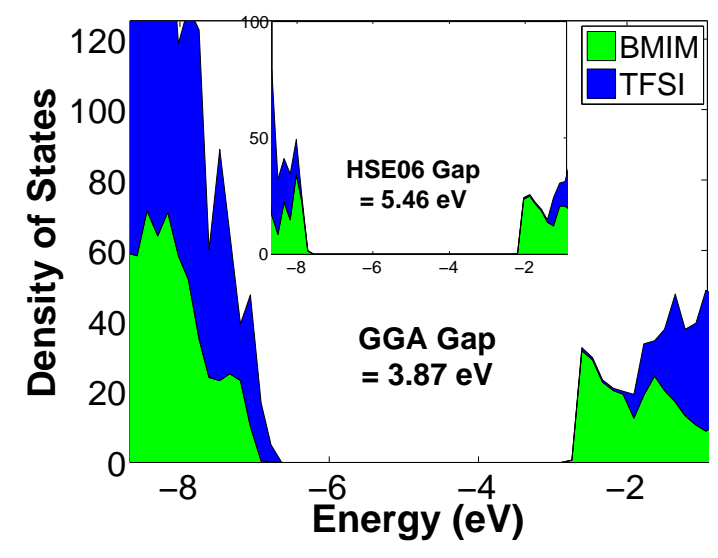

(e) BMIM TFSI

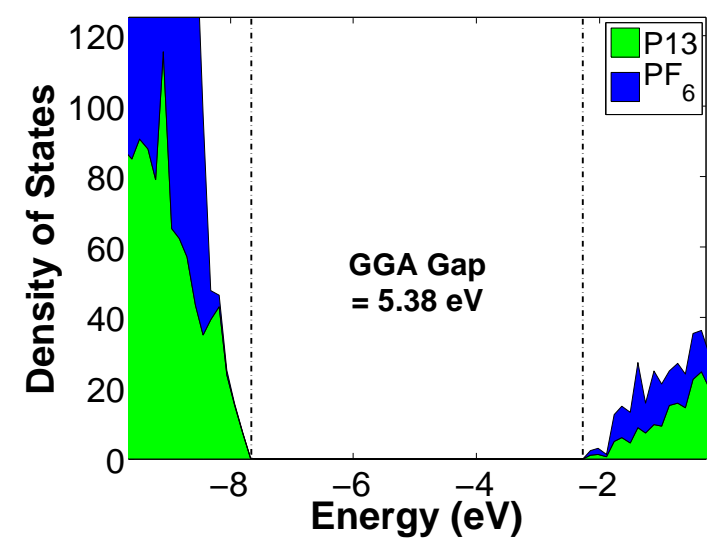

(b) $\mathrm{P} 13 \mathrm{PF}_{6}$

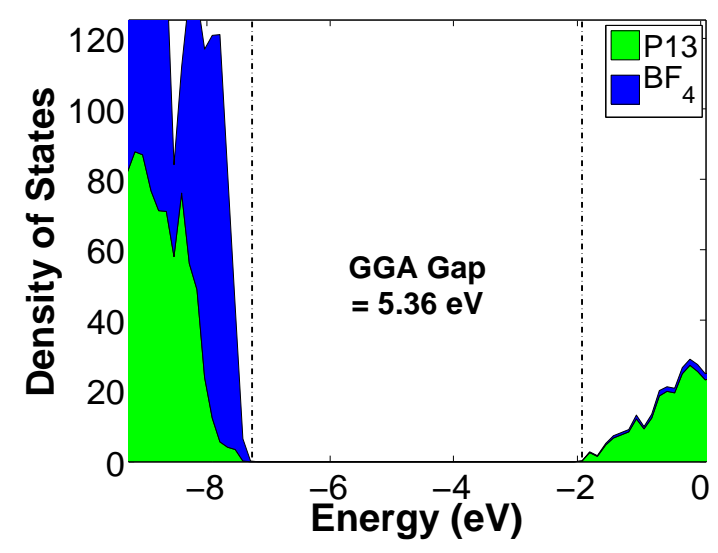

(d) $\mathrm{P} 13 \mathrm{BF}_{4}$

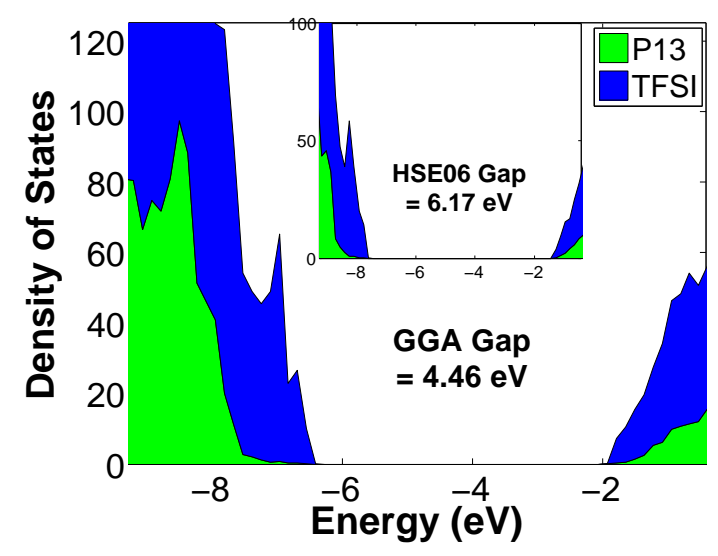

(f) P13 TFSI

Figure 3: Representative density of states for the six ILs investigated calculated using GGA, decomposed by cation and anion contributions. For the four experimentally known ILs, the insets show the HSE06 density of states and gaps for comparison. 
significant contributions from both $\mathrm{P} 13$ and $\mathrm{PF}_{6}$ in the LUMO, which would imply that $\mathrm{PF}_{6}$ could contribute to the reductive stability when paired with P13 as well. This is surprising, given than $\mathrm{PF}_{6}$ is typically found to be the most stable anion among the three studied.

3. Anodic limits of TFSI-based ILs and P13 $\mathbf{B F}_{4}$ For TFSI-based ILs and P13 BF 4 , the HOMOs are always dominated by anion states, which implies that the anodic stability is set by the anion.

4. Evidence of possible cation anodic instability. For $\mathrm{BMIM} \mathrm{BF}_{4}, \mathrm{BMIM} \mathrm{PF}_{6}$ and $\mathrm{P} 13 \mathrm{PF}_{6}$, the HOMO is dominated by cation states, which implies that the oxidation stability is no longer limited by the anion, but rather by the stability of the cation. This observation challenges the prevailing assumption that it is always the anion that determines oxidative stability. To our knowledge, there is no experimental evidence pointing to possible cation oxidative instability in ILs thus far. The possible anodic instability of BMIM would also explain the earlier observation that regardless of the variation in the anionic species, all BMIM-based ILs have relatively similar electrochemical windows. Because both the HOMO and LUMO states of $\mathrm{BMIM} \mathrm{PF}_{6}$ and $\mathrm{BMIM} \mathrm{BF}_{4}$ are dominated by cation states, we would expect the cation to set the electrochemical window. For BMIM TFSI, the LUMO states are dominated by the TFSI anion, but the cation states are only slightly lower in energy. We note that the HSE06 functional leads to largely similar results as GGA, but predicts the LUMO states to be dominated by BMIM instead of TFSI for BMIM TFSI (see insets in Figure 3).

\section{Vacuum and PCM electrochemical windows}

Figure 4 and Figure 5 show the calculated cathodic and anodic limits for all ions investigated using vacuum and PCM calculations respectively. For each IL formed from a particular cation-anion pair, the overall cathodic limit is set by the maximum of cathodic limits of its constituent ions, and the overall anodic limit is set by the minimum of the anodic limit of its constituent ions. To take $\mathrm{BMIM} \mathrm{PF}_{6}$ as an example, the cathodic and anodic limits in vacuo are set by the BMIM cation 
and $\mathrm{PF}_{6}$ anion respectively, while the $\mathrm{PCM}$ cathodic and anodic limits are both set by the BMIM cation.

From Figure 4, we may observe that the vacuum calculations predict limits that are effectively set by respective ions, i.e., cathodic limits by cations and anodic limits by anions. On the other hand, the PCM calculations predict limits (cathodic and anodic) that are mostly set by the cations. Even $\mathrm{P} 13$ is predicted to have a lower anodic stability than the $\mathrm{BF}_{4}$ and $\mathrm{PF}_{6}$ anions. The significant stabilization from the isotropic dielectric response is evident from the significantly wider electrochemical windows (anodic limit - cathodic limit) in the PCM calculations compared to the vacuum calculations. Electrochemical windows in excess of $6 \mathrm{~V}$ are obtained, which are much wider than the typical 4-6 V observed experimentally for these ILs. This observation indicates that the PCM model may have over-stabilized the ions.

We note that recent solvatochrmonic measurements ${ }^{82}$ as well as computer simulations ${ }^{83-85}$ have found that the effective solvent polarity of ILs is much higher than suggested by their dielectric constant. In particular, Reichardt et al. ${ }^{82}$ have demonstrated that the effective solvent polarity of ILs, as measured by the solvatochromic absorption of the zwitterionic betaine dye 2,6-diphenyl4-(2,4,6-triphenylpyridinium-1-yl)phenolate dye $E_{T}(30)$, is comparable to that of molecular dipolar non-hydrogen bond donor (HBD) and dipolar HBD solvents. Hence, even though there is no simple mapping of the $E_{T}(30)$ solvent polarity scale to the necessary dielectric constant parameter required for PCM calculations, we performed PCM calculations using a higher dielectric constant of 30 to ascertain the effect of the dielectric constant on the predicted cathodic and anodic limits (see the Supporting Information). We found that some of the calculated electrochemical windows are slightly smaller (e.g., the electrochemical window of $\mathrm{BMIM} \mathrm{PF}_{6}$ decreased from $6.51 \mathrm{~V}$ to $6.34 \mathrm{~V}$ ) when a higher dielectric constant of 30 is used, due to the greater dielectric stabilization effect for more highly-charged ions. Nonetheless, the general observation that PCM calculations significantly overestimate the electrochemical windows still holds. 


\section{TFSI}
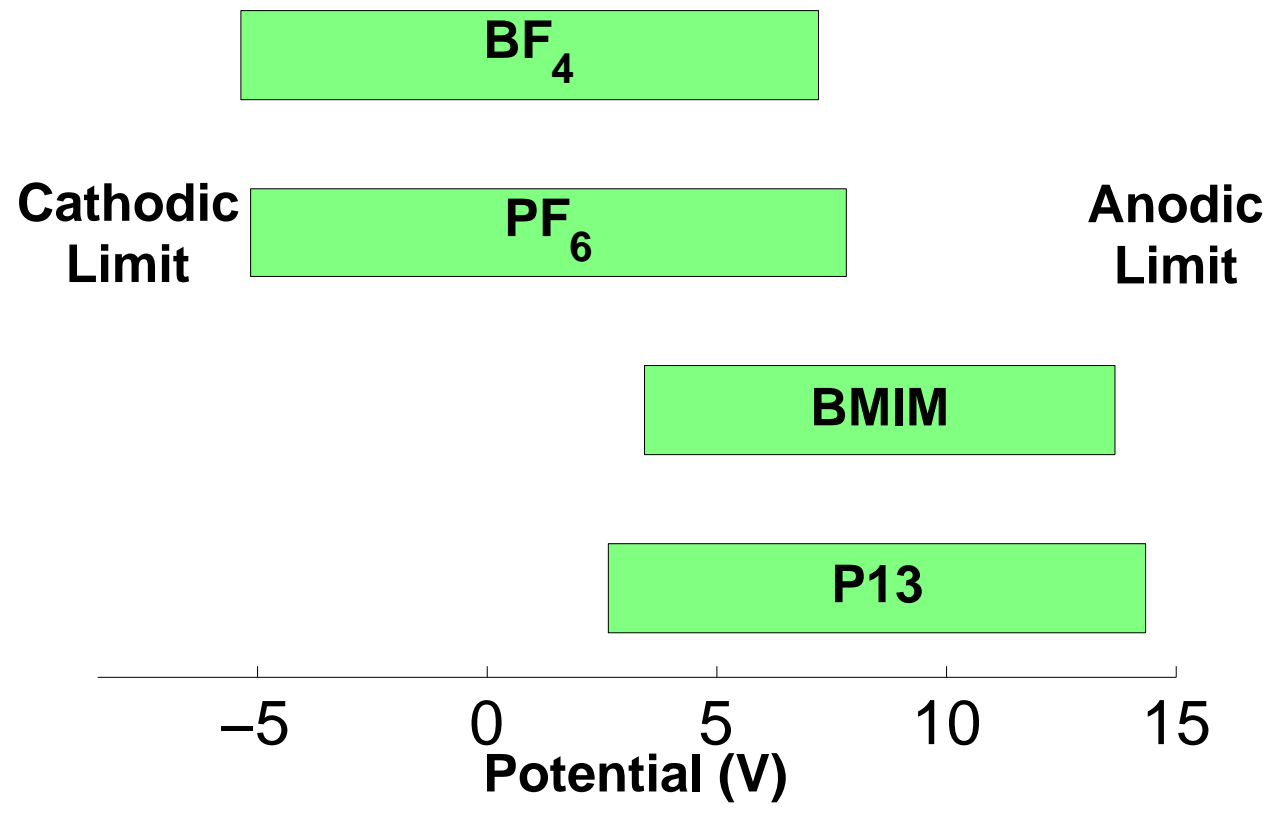

Figure 4: Calculated cathodic and anodic limits for individual ions using isolated molecule calculations at the B3LYP/6-31+G(d)//B3LYP/6-311+G(2d,p) level. 


\section{TFSI}

\section{BF $_{4}$}

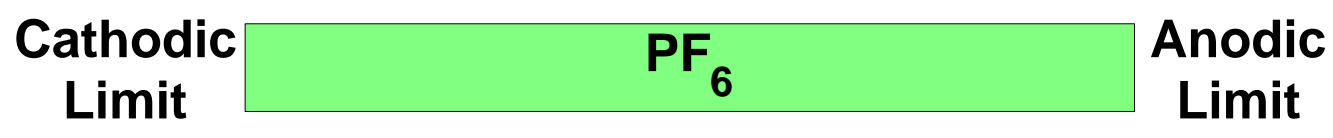

\section{BMIM}

\section{P13}

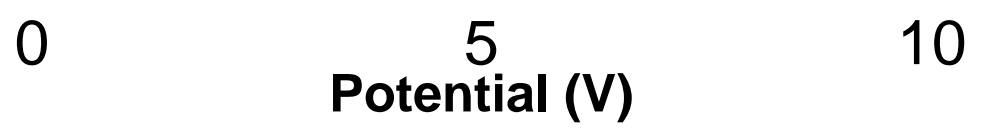

Figure 5: Calculated cathodic and anodic limits for individual ions using PCM calculations at the B3LYP/6-31+G(d)//B3LYP/6-311+G(2d,p) level with a dielectric constant of 12. 


\section{Discussion}

Table 2: Summary of the electrochemical windows $(E W)$, cathodic limit $\left(V_{\mathrm{CL}}\right)$ and anodic limit $\left(V_{\mathrm{AL}}\right)$ for the six ILs determined using the three levels of approximations. The letter in () denotes the major contributing species to the limit, where $\mathrm{C}$ stands for cation and A stands for anion.

\begin{tabular}{lccccccccc}
\hline Ionic & \multicolumn{2}{c}{$\mathrm{MD}+\mathrm{PB}$ DFT (V) } & \multicolumn{3}{c}{$\mathrm{PCM}(\mathrm{V})$} & \multicolumn{3}{c}{ In vacuo (V) } \\
Liquid & $V_{\mathrm{CL}}$ & $V_{\mathrm{AL}}$ & $E W$ & $V_{\mathrm{CL}}$ & $V_{\mathrm{AL}}$ & $E W$ & $V_{\mathrm{CL}}$ & $V_{\mathrm{AL}}$ & $E W$ \\
\hline BMIM PF $_{6}$ & $3.16(\mathrm{C})$ & $6.75(\mathrm{C})$ & 3.59 & $1.69(\mathrm{C})$ & $8.20(\mathrm{C})$ & 6.51 & $3.44(\mathrm{C})$ & $7.83(\mathrm{~A})$ & 4.39 \\
BMIM BF $_{4}$ & $3.29(\mathrm{C})$ & $6.92(\mathrm{C})$ & 3.63 & $1.69(\mathrm{C})$ & $8.20(\mathrm{C})$ & 6.51 & $3.44(\mathrm{C})$ & $7.21(\mathrm{~A})$ & 3.77 \\
BMIM TFSI & $3.01(\mathrm{C})$ & $6.63(\mathrm{~A})$ & 3.62 & $1.69(\mathrm{C})$ & $8.20(\mathrm{C})$ & 6.51 & $3.44(\mathrm{C})$ & $6.69(\mathrm{~A})$ & 3.25 \\
P13 PF $_{6}$ & $2.21(\mathrm{C} / \mathrm{A})$ & $7.69(\mathrm{C})$ & 5.48 & $0.82(\mathrm{C})$ & $9.16(\mathrm{C})$ & 8.34 & $2.64(\mathrm{C})$ & $7.83(\mathrm{~A})$ & 5.19 \\
P13 BF $_{4}$ & $1.97(\mathrm{C})$ & $7.17(\mathrm{~A})$ & 5.20 & $0.82(\mathrm{C})$ & $9.16(\mathrm{C})$ & 8.34 & $2.64(\mathrm{C})$ & $7.21(\mathrm{~A})$ & 4.57 \\
P13 TFSI & $2.06(\mathrm{~A})$ & $6.38(\mathrm{~A})$ & 4.32 & $0.82(\mathrm{C})$ & $8.34(\mathrm{~A})$ & 7.52 & $2.64(\mathrm{C})$ & $6.69(\mathrm{~A})$ & 4.05 \\
\hline
\end{tabular}

Table 2 summarizes the cathodic and anodic limits of the six ILs obtained from the three levels of approximations. We found that in vacuo calculations, which do not take into account any effect of the local environment in solution on the ion stability, results in limits that are effectively set by respective ions, i.e., cathodic limits by cations and anodic limits by anions. While such calculations may be used to ascertain broad general trends in the relative stability of different ions (e.g., $\mathrm{P} 13$ versus $\mathrm{BMIM}$ or $\mathrm{PF}_{6}$ versus $\mathrm{BF}_{4}$ versus TFSI), they are insufficient to provide quantitative accuracy.

The cathodic and anodic limits from the PCM calculations show a significant stabilization effect from the dielectric response, resulting in electrochemical windows that are significantly wider than the vacuum calculations. This stabilization is likely to be overestimated; in real ILs, the dielectric effect is likely to be highly anisotropic and highly dependent on the coordination of the first solvent shell of counter-ions. Hence, the value of the PCM in modeling ILs appears to be limited, especially since the PCM requires the dielectric constant as an external input, which might not be available for less-studied IL systems.

We believe our approach of using a combination of MD and DFT calculations with a Hartree potential alignment procedure provide the most accurate model of an IL among the three level of approximations considered. The overall liquid structure of an IL is explicitly modeled, and ion-ion interactions are taken into account. This approach can potentially be applied to the study of other 
liquid systems. The main drawback is the need to find or develop force fields that capture the essential physics of the liquid structure in order to perform the MD simulations. While this drawback would not be a major issue for well-studied systems such as organic solvents, it could prove to be a significant hurdle when studying novel systems. However, we note that specifically for ILs, transferable force fields have been developed that can describe bulk and interfacial properties of a wide set of ILs accurately. ${ }^{86}$

Given the difficulties in comparing experimental data on electrochemical windows and the inherent limitations of DFT in characterizing unoccupied states, it is difficult for us to make a definitive statement on the accuracy of our calculations. Nevertheless, our calculations are in broad agreement with experimental data, and have provided crucial insights into the species limiting the redox stability of the solvent. Our calculations predict the cathodic stability of the TFSI anion to be limiting in the case of P13 TFSI, in agreement with previous experiments and calculations. ${ }^{1}$ In addition, our work predicts the anodic stability of the cation to be a limiting factor in $\mathrm{BMIM} \mathrm{PF}_{6}$, BMIM BF 4 and $\mathrm{P}_{13} \mathrm{PF}_{6}$. In particular, the relative instability of the BMIM anion toward oxidation could account for the similar measured electrochemical windows for BMIM-based ILs; the BMIM cation could be limiting at both the anodic and cathodic limits.

To our knowledge, no experimental or theoretical work thus far have shown any indication of possible cation anodic instability. This "gap" in the literature is particularly puzzling to us as there is experimental evidence in the literature that points to possible cation anodic instability. For example, Buzzeo et al. ${ }^{14}$ measured 1-ethyl-3-methyl imidazolium (EMIM) TFSI and 1-hexyl-3methyl imidazolium (HMIM) trifluorotris-(pentafluoro-ethyl)phosphate (FAP) to have very similar anodic limits, despite the fact that FAP is expected to be significantly more anodically stable than the TFSI anion. Though none of these specific ions with the exception of TFSI were investigated in our work, our results suggest that the imidazolium-based cations (EMIM and HMIM) could possibly be the limiting species at the anodic limits for these ILs. It is our wish that this prediction would be verified by future experimental work. For instance, Kroon et al. ${ }^{87}$ previously conducted an extensive theoretical and experimental investigation into the decomposition pathways and prod- 
ucts for N-N-butylmethylpyrrolidinium TFSI and BMIM BF 4 ILs on the cathode limit and showed that the predicted decomposition products are in excellent agreement with experiments. However, we have found no similar investigations on the anodic limit in the literature.

Our work therefore highlights that the prevalent assumption that the cation sets the cathodic limit and the anion sets the anodic limits may not be valid for a significant number of ILs. This could have implications in the design of ILs for electrochemical applications in that it is no longer sufficient to optimize the respective ion for the respective limits; the cathodic and anodic limits of both cation and anion must be taken into account.

\section{Conclusion}

In this work, we investigated the cathodic and anodic limits of six room-temperature ionic liquids (ILs) formed from the BMIM and P13 cations, and the $\mathrm{PF}_{6}, \mathrm{BF}_{4}$ and TFSI anions using a combination of MD simulations and DFT calculations. In our approach, all inter-ion interactions were taken into account by explicitly modeling the entire liquid structure. The relative cathodic and anodic limits of $\mathrm{BMIM} \mathrm{PF}_{6}, \mathrm{BMIM} \mathrm{BF}_{4}, \mathrm{BMIM}$ TFSI and P13 TFSI obtained from our approach are in fairly good agreement with existing experimental data. The cation- and anion-projected DOSs obtained not only predict the TFSI anion to be less stable than the P13 cation against reduction (in agreement with previous findings ${ }^{1}$ ), but also provide some surprising evidence of possible cation anodic instability. We predict the aromatic BMIM cation to be less stable against oxidation than the respective anions in $\mathrm{BMIM} \mathrm{PF}_{6}$ and $\mathrm{BMIM} \mathrm{BF}_{4}$, and the $\mathrm{P} 13$ cation to be less stable against oxidation than the $\mathrm{PF}_{6}$ anion in $\mathrm{P}_{13} \mathrm{PF}_{6}$. We also compared the predictions of our approach with that of simpler approximations based on the calculated electron affinities and ionization energies of isolated ions using in vacuo or PCM calculations. We found that the PCM overstabilized the ions, resulting in predicted electrochemical windows that are much larger than those measured in experiments, while in vacuo calculations are insufficient to achieve quantitative accuracy due to the lack of local environment effects. 


\section{Acknowledgement}

This work was supported by E. I. du Pont de Nemours \& Co. through the DuPont-MIT Alliance program. The authors would like to thank William L. Holstein and Steve R. Lustig from DuPont for their useful insights and assistance. This research was supported in part by the National Science Foundation through TeraGrid resources provided by Pittsburgh Supercomputing Center.

\section{Supporting Information Available}

The Hartree potential plots for the room-temperature ionic liquids investigated are available free of charge via the Internet at http://pubs.acs.org.

\section{References}

[1] Howlett, P. C.; Izgorodina, E. I.; Forsyth, M.; Macfarlane, D. R. Zeitschrift fur Physikalische Chemie 2006, 220, 1483-1498.

[2] Xu, K. Chemical Reviews 2004, 104, 4303-4418.

[3] McEwen, A. B.; McDevitt, S. F.; Koch, V. R. Journal of The Electrochemical Society 1997, 144, L84-L86.

[4] McEwen, A. B.; Ngo, H. L.; LeCompte, K.; Goldman, J. L. Journal of The Electrochemical Society 1999, 146, 1687-1695.

[5] Lewandowski, A.; Galinski, M. Journal of Physics and Chemistry of Solids 2004, 65, 281286.

[6] Garcia, B.; Lavallée, S.; Perron, G.; Michot, C.; Armand, M. Electrochimica Acta 2004, 49, 4583-4588.

[7] Matsumoto, H.; Matsuda, T.; Miyazaki, Y. Chemistry Letters 2000, 1430-1431. 
[8] Nakagawa, H.; Izuchi, S.; Kuwana, K.; Nukuda, T.; Aihara, Y. Journal of The Electrochemical Society 2003, 150, A695-A700.

[9] Sakaebe, H.; Matsumoto, H.; Tatsumi, K. Electrochimica Acta 2007, 53, 1048-1054.

[10] Galinski, M.; Lewandowski, A.; Stepniak, I. Electrochim. Acta 2006, 51, 5567-5580.

[11] Hapiot, P.; Lagrost, C. Chemical Reviews 2008, 108, 2238-2264.

[12] Zhou, F.; Cococcioni, M.; Kang, K.; Ceder, G. Electrochem. Commun. 2004, 6, 1144-1148.

[13] Ma, X.; Kang, B.; Ceder, G. Journal of The Electrochemical Society 2010, 157, A925.

[14] Buzzeo, M.; Hardacre, C.; Compton, R. ChemPhysChem 2006, 7, 176 - 180.

[15] Rogers, E. I.; SÃČC̃ěljukic ÃČ̃̃ ̌̌, B.; Hardacre, C.; Compton, R. G. Journal of Chemical \& Engineering Data 2009, 54, 2049-2053.

[16] Prendergast, D.; Grossman, J. C.; Galli, G. Journal of Chemical Physics 2005, 123, 014501.

[17] Koch, V.; Dominey, L.; Nanjundiah, C. Journal of the Electrochemical Society 1996, 143, 798-803.

[18] Ue, M.; Murakami, A.; Nakamura, S. Journal of the Electrochemical Society 2002, 149, $1572-1577$.

[19] Vollmer, J. M.; Curtiss, L. A.; Vissers, D. R.; Amine, K. Journal of The Electrochemical Society 2004, 151, A178-A183.

[20] Zhang, S.; Sun, N.; He, X.; Lu, X.; Zhang, X. Green Chemistry 2006, 35, 1475.

[21] Tasaki, K.; Kanda, K.; Kobayashi, T.; Nakamura, S.; Ue, M. Journal of the Electrochemical Society 2006, 153, 2192-2197.

[22] Johansson, P. Physical Chemistry Chemical Physics 2007, 9, 1493-1498. 
[23] Ong, S. P.; Ceder, G. Electrochimica Acta 2010, 55, 3804-3811.

[24] Maeshima, H.; Moriwake, H.; Kuwabara, A.; Fisher, C. a. J. Journal of The Electrochemical Society 2010, 157, A696.

[25] Ngo, H.; LeCompte, K.; Hargens, L.; McEwen, A. Thermochimica Acta 2000, 357, 97-102.

[26] Martin, R. M. Electronic Structure: Basic Theory and Practical Methods (Vol 1); Cambridge University Press, 2004; p 648.

[27] Tomasi, J.; Cammi, R.; Mennucci, B.; Cappelli, C.; Corni, S. Physical Chemistry Chemical Physics 2002, 4, 5697-5712.

[28] Tomasi, J.; Mennucci, B.; Cammi, R. Chemical Reviews 2005, 105, 2999-3093.

[29] Cossi, M.; Rega, N.; Scalmani, G.; Barone, V. Journal of Chemical Physics 2001, 114, 5691.

[30] Cossi, M.; Scalmani, G.; Rega, N.; Barone, V. Journal of Chemical Physics 2002, 117, 43.

[31] Cossi, M.; Barone, V. Journal of Chemical Physics 2000, 112, 2427.

[32] Cossi, M.; Barone, V. Journal of Chemical Physics 2001, 115, 4708.

[33] Cossi, M.; Barone, V.; Robb, M. A. Journal of Chemical Physics 1999, 111, 5295.

[34] Cossi, M.; Barone, V. Journal of Physical Chemistry A 2000, 104, 10614-10622.

[35] Cammi, R.; Mennucci, B.; Tomasi, J. Journal of Physical Chemistry A 1999, 103, 9100-9108.

[36] Cammi, R.; Mennucci, B.; Tomasi, J. Journal of Physical Chemistry A 2000, 104, 5631-5637.

[37] Mennucci, B.; Tomasi, J. Journal of Chemical Physics 1997, 106, 5151.

[38] Mennucci, B.; Cancès, E.; Tomasi, J. Journal of Physical Chemistry B 1997, 101, 1050610517.

[39] Barone, V.; Cossi, M.; Tomasi, J. Journal of Computational Chemistry 1998, 19, 404-417. 
[40] Barone, V.; Cossi, M. Journal of Physical Chemistry A 1998, 102, 1995-2001.

[41] Cossi, M.; Barone, V.; Mennucci, B.; Tomasi, J. Chemical Physics Letters 1998, 286, 253260.

[42] Barone, V.; Cossi, M.; Tomasi, J. Journal of Chemical Physics 1997, 107, 3210.

[43] Cance Ã ̌̌̃̃̃̌s, E.; Mennucci, B.; Tomasi, J. Journal of Chemical Physics 1997, 107, 3032.

[44] Cossi, M.; Barone, V.; Cammi, R.; Tomasi, J. Chemical Physics Letters 1996, 255, 327-335.

[45] Miertus, S.; Tomasi, J. Chemical Physics 1982, 65, 239-245.

[46] Miertuš, S.; Scrocco, E.; Tomasi, J. Chemical Physics 1981, 55, 117-129.

[47] Smith, W.; Yong, C.; Rodger, P. Molecular Simulation 2002, 28, 385-471.

[48] Smith, W.; Forester, T. R. Journal of Molecular Graphics 1996, 14, 136-141.

[49] Smith, W. Molecular Simulation 2006, 32, 933-933.

[50] Jorgensen, W. L.; Maxwell, D. S.; Tirado-Rives, J. Journal of the American Chemical Society 1996, $118,11225-11236$.

[51] Canongia Lopes, J. N.; Pádua, A. A. H. Journal of Physical Chemistry B 2004, 108, 1689316898.

[52] Canongia Lopes, J. N.; Deschamps, J.; Pádua, A. A. H. Journal of Physical Chemistry B 2004, 108, 11250-11250.

[53] Canongia Lopes, J. N.; Deschamps, J.; Pádua, A. A. H. Journal of Physical Chemistry B 2004, 108, 2038-2047.

[54] Breneman, C. M.; Wiberg, K. B. Journal of Computational Chemistry 1990, 11, 361-373.

[55] Berendsen, H. J. C.; Postma, J. P. M.; van Gunsteren, W. F.; DiNola, A.; Haak, J. R. Journal of Chemical Physics 1984, 81, 3684. 
[56] Kresse, G.; Joubert, D. Physical Review B 1999, 59, 1758-1775.

[57] Kresse, G.; Furthmuller, J. Physical Review B 1996, 54, 11169-11186.

[58] Mrovec, M.; Albina, J.-M.; Meyer, B.; Elsässer, C. Physical Review B 2009, 79, 245121.

[59] Alkauskas, A.; Broqvist, P.; Devynck, F.; Pasquarello, A. Physical Review Letters 2008, 101, 106802.

[60] Shaltaf, R.; Rignanese, G.-M.; Gonze, X.; Giustino, F.; Pasquarello, A. Physical Review Letters 2008, 100, 186401.

[61] Frisch, M. J. et al. Gaussian 03, Revision C.02.

[62] Becke, A. Journal of Chemical Physics 1993, 98, 5648-5652.

[63] Lee, C. T.; Yang, W. T.; Parr, R. G. Physical Review B 1988, 37, 785-789.

[64] Curtiss, L. A.; Redfern, P. C.; Raghavachari, K.; Pople, J. A. Journal of Chemical Physics 1998, $109,42$.

[65] Scott, A. P.; Radom, L. Journal of Physical Chemistry 1996, 100, 16502-16513.

[66] Weingartner, H. Zeitschrift fur Physikalische Chemie 2006, 220, 1395-1405.

[67] Lide, D. R., Ed. CRC Handbook of Chemistry and Physics, Internet Version 2011, 91st ed.; CRC Press, 2010; pp 12-114.

[68] Fitchett, B.; Rollins, J.; Conboy, J. Journal of the Electrochemical Society 2004, 151, E219E225.

[69] Randström, S.; Montanino, M.; Appetecchi, G.; Lagergrenb, C.; Morenoa, A.; Passerini, S. Electrochimica Acta 2008, 53, 6397-6401.

[70] OâǍŽÃ ̌̃̃̃t'Mahony, A. M.; Silvester, D. S.; Aldous, L.; Hardacre, C.; Compton, R. G. Journal of Chemical and Engineering Data 2008, 53, 2884-2891. 
[71] Lewandowski, A.; Stepniak, I. Physical Chemistry Chemical Physics 2003, 5, 4215.

[72] Cohen, A.; Mori-Sánchez, P.; Yang, W. Physical Review B 2008, 77.

[73] Sham, L.; Schlüter, M. Physical Review Letters 1983, 51, 1888-1891.

[74] Wang, C.; Pickett, W. Physical Review Letters 1983, 51, 597-600.

[75] Chan, M.; Ceder, G. Physical Review Letters 2010, 105, 196403.

[76] Heyd, J.; Scuseria, G. E.; Ernzerhof, M. Journal of Chemical Physics 2003, 118, 8207.

[77] Heyd, J.; Scuseria, G. E.; Ernzerhof, M. Journal of Chemical Physics 2006, 124, 219906.

[78] Heyd, J.; Scuseria, G. E. Journal of Chemical Physics 2004, 121, 1187-92.

[79] Lewandowski, A.; Swiderska-Mocek, A. Journal of Applied Electrochemistry 2010, 40, 515524.

[80] Barisci, J.; Wallace, G.; MacFarlane, D.; Baughman, R. Electrochemistry Communications 2004, 6, 22-27.

[81] Fernicola, A.; Croce, F.; Scrosati, B.; Watanabe, T.; Ohno, H. Journal of Power Sources 2007, $174,342-348$.

[82] Reichardt, C. Green Chemistry 2005, 7, 339.

[83] Shim, Y.; Duan, J.; Choi, M. Y.; Kim, H. J. The Journal of Chemical Physics 2003, 119, 6411.

[84] Shim, Y.; Choi, M. Y.; Kim, H. J. The Journal of chemical physics 2005, 122, 44510.

[85] Shim, Y.; Choi, M. Y.; Kim, H. J. The Journal of chemical physics 2005, 122, 44511.

[86] Borodin, O. Journal of Physical Chemistry B 2009, 113, 11463-78.

[87] Kroon, M.; Buijs, W.; Peters, C.; Witkamp, G.-J. Green Chemistry 2006, 8, 241-245. This material is available free of charge via the Internet at http://pubs acs.org/. 